\title{
Testing primates with joystick-based automated apparatus: Lessons from the Language Research Center's Computerized Test System
}

\author{
DAVID A. WASHBURN and DUANE M. RUMBAUGH \\ Sonny Carter Life Sciences Laboratory, Georgia State University, Atlanta, Georgia
}

Nonhuman primates provide useful models for studying a variety of medical, biological, and behavioral topics. Four years of joystick-based automated testing of monkeys using the Language Research Center's Computerized Test System (LRC-CTS) are examined to derive hints and principles for comparable testing with other species-including humans. The results of multiple parametric studies are reviewed, and reliability data are presented to reveal the surprises and pitfalls associated with video-task testing of performance.

Nonhuman primates are critical to many areas of scientific inquiry, providing an important model for the study of physiology, biomedicine, and psychology across species. In the present report, 4 years of research with rhesus monkeys (Macaca mulatta) are examined to derive principles and caveats for joystick-based, automated testing with a variety of primate species-including humans. It is anticipated that the practical lessons gleaned from this experience will provide useful information for researchers establishing a computerized testing environment.

\section{A CASE STUDY}

In the spring of 1987 , a project was initiated at Georgia State University to develop a new testing paradigm for research and environmental enrichment with rhesus monkeys (Macaca mulatta) as subjects. Project scientists chose to abandon existing technology requiring simple operant responses to basic stimuli and to avoid the labor-intensivealbeit effective, flexible, and standard-Wisconsin General Test Apparatus and its revisions. The model that was selected for development, used in research with humans and apes, and borrowed from computer gaming technology, required the manipulation of a joystick in response to computer-generated and -controlled stimuli. The test system that we developed is called the Language Research Center's Computerized Test System (LRC-CTS).

This research was supported in part by Grant NAG2-438 from the National Aeronautics and Space Administration to Georgia State University and by HD-06016 from the National Institutes of Health. Additional support was provided by the College of Arts and Sciences of Georgia State University. The authors wish to acknowledge theoretical and technical contributions to this effort by William D. Hopkins, W. Kirk Richardson, and E. Sue Savage-Rumbaugh. Preparation of this paper was facilitated by use of the Allan M. Schrier Commemorative Library of the Language Research Center, Georgia State University. Correspondence concerning this article should be sent to David A. Washburn, Department of Psychology, Georgia State University, Atlanta, GA 30303.
Previous research had demonstrated that languagetrained chimpanzees could learn by observation to manipulate a joystick appropriate to computer-generated conditions (Savage-Rumbaugh, 1986). However, in initial experiments, two relatively naive chimpanzees failed to acquire these skills. This finding, combined with a substantial literature demonstrating that rhesus monkeys fail to learn when salient stimuli are spatially discontiguous from response loci (Meyer, Treichler, \& Meyer, 1965; Stollnitz, 1965), suggested that gains to be made with the rhesus along these lines would be slow and arduous at best. In point of fact, the venture was seen even by project scientists as at high risk for failure, but as an effort with limitless potential under conditions of reasonable success.

To date, 10 rhesus monkeys at our laboratory, as well as several dozen animals at other laboratories, have learned, using the LRC-CTS, to respond in a variety of computer-generated tasks by skillfully manipulating a joystick. Most of the details of initial training were first discussed elsewhere (Rumbaugh, Richardson, Washburn, Savage-Rumbaugh, \& Hopkins, 1989; Washburn, Hopkins, \& Rumbaugh, 1989), and many technical considerations regarding the LRC-CTS (hardware and software) have been reviewed (Richardson, Washburn, Hopkins, Savage-Rumbaugh, \& Rumbaugh, 1990). Our purpose in the present paper is to provide general guidelines for implementing joystick-based automated test stations for testing human and nonhuman primates by reviewing the parametric findings and reliability data produced over the previous 4 years of LRC-CTS use.

\section{SUBJECTS}

It must be emphasized that the test system advanced in this review has proved effective for comparative and cognitive testing with human and a variety of nonhuman primates. Data from the two rhesus monkeys initially trained (Abel and Baker) have been replicated with numerous other representatives of the species. Orangutans (Pongo), 
chimpanzees (Pan troglodytes and paniscus), and humans (both normally developing and mentally retarded children, as well as adults) have also been tested fruitfully with this paradigm (see, e.g., Hopkins, Washburn, \& Rumbaugh, 1990; Smith, Cash, Barr, \& Putney, 1986; Washburn \& Rumbaugh, 1991). Encouraging data from other species are emerging from other laboratories. Although many of the guidelines provided in the present review will be tailored for applications with rhesus monkeys, they do not impugn the attempt to use the system with other species.

\section{APPARATUS}

The current configuration of apparatus integrated into the test stations remains fairly faithful to details of the LRC-CTS provided earlier (Richardson et al., 1990). IBM-compatible platforms from a variety of manufacturers are currently employed; they range from simple $8-\mathrm{MHz}, 8088$-based XT systems up to $16-\mathrm{MHz}, 386 \mathrm{SX}$ computers. Although the video capabilities of these systems cover the range of widely available formats, current software has been written for compatibility with the lowest common denominator of color video display (CGA) and will in fact work with systems utilizing monochrome graphics boards.

Each computer is equipped with a 12-channel digital I/O board (see Appendix A) connected to a solid-state relay board via a 37-pin ribbon cable. This controls the delivery of pellets in a pellet dispenser, which operates on ac power with an optional adapter. Several pellet sizes have been used (see discussion below), and conversion kits for changing the dispenser for new pellet sizes are also available. Pellets are allowed to fall through tubing into a cup accessible to the subject.

For the joystick to work reliably, given the range of potential computer processor speeds, a programmable game board such as the Kraft Multispeed Programmable Joystick Adapter Card should be used. This card, which can be calibrated for the speed of the computer (to $25 \mathrm{MHz}$ ), reduces much of the error that accompanies joystick use. Practically any IBM-compatible, spring-centered analog joystick can be used with the system. No substantial differences in quality or durability have been observed, despite a price range from $\$ 15$ to over $\$ 100$.

Although definitive data are not available at this point, it is anticipated that sound feedback emanating from the general direction of the monitor (rather than the computer, which may be located at some distance) and at sufficient volume to be heard clearly is critical for ease of learning in primates. Thus, although there is substantial evidence that rhesus and other species can respond with minimal disruption in the absence of sound feedback after they have been trained, proper control of computer-generated sound may be essential for initial training. Such control is also convenient for other purposes (e.g., testing humans). The modifications necessary to reroute computer-generated sound from the internal speaker to an external speaker amplifier have been provided in detail elsewhere (Washburn, 1992b).

\section{PROCEDURE}

At our laboratory, animals are tested at dedicated computer stations that are secured to the walls and are protected within Lexan enclosures. Each successful trial is rewarded with one or more $97-\mathrm{mg}$ fruit-flavored chow pellets. Given this arrangement, animals can be given access to the test system for 16-24 h each day, and they thereby provide themselves with most or all of their daily nutritive intake. The animals work ad lib within each workday, and they are not restrained within their home cages, reduced in body weight, or deprived of water. Supplemental chow is provided daily when necessary.

Each animal is trained on a battery of 18 computerized tasks (Appendix B). These tasks have been described elsewhere and will not be discussed in detail here. The tasks are organized into a curriculum, with many tasks establishing requisite skills for the subsequent ones. Training begins with relatively simple shaping tasks and advances to complex learning, memory, and problem-solving tasks. Animals are moved from task to task on the basis of performance criteria that have been empirically established. At several points within the task progression, however, the experimenter can branch off into specific subsets of skills or paradigms.

\section{CASE STUDY CONTINUED}

In preparing the present paper, we reviewed the communications and activities from 1987 to determine what were and what were not the principal concerns when we began the project. We compared these concerns with the records of actual progress and of problems that have arisen. We discovered that a great many problems were anticipated that never materialized; other important issues emerged that were relatively unanticipated.

\section{Could Rhesus Monkeys Learn to Manipulate the Joystick Appropriately?}

As we have already mentioned, there was substantial concern that rhesus monkeys could not learn to respond in ways that were appropriate and necessary for the effective use of the LRC-CTS. Knowledge that manipulations of the joystick were faithfully reflected as movements of a cursor on screen-that, in effect, the cursor represented actions-was believed to be beyond macaque competencies. Notwithstanding, the animals have without exception and with substantial facility acquired the requisite skills for LRC-CTS performance.

A shaping program (SIDE; see Rumbaugh et al., 1989) was designed to train monkeys to move the joystick. The animals were required to discriminate increasingly finer stimulus loci and to exercise increasingly greater motor precision. Once the animals could convincingly bring the cursor into contact with a small, stationary target, the target was set in motion (CHASE task). Thus, the ability to bring the cursor into contact with a moving target (relative to some criterion level) operationally marks the transition between "signaled directional leverpressing with 
a joystick" and skillful joystick control that reflects movements of the cursor on screen as an extension, of sorts, of the subject's hand. To date, no rhesus monkeys have been tested to determine whether this transition would $\alpha c$ cur in the absence of SIDE or some similar shaping procedure; that is, rhesus monkeys have not shown (or been given the opportunity to show) evidence of observational learning of joystick skills as have chimpanzees. However, rhesus's learning curves using the SIDE task are impressive in numerous ways.

The animals trained at our laboratory have progressed through the seven criterion-based stages of the SIDE task and have reached a rather stringent criterion on the CHASE task in an average of under 5,000 trials. Given the work rates of the animals, this can reflect only days or (at most) several weeks of training. Current data also suggest that reaching criterion in the SIDE task is the most demanding and time-consuming portion of training on any task. That is, each subsequent complex task in the battery (e.g., pursuit tracking, learning set, matching to sample, maze solving) can be taught relatively easily and quickly after SIDE and CHASE have been mastered. In addition, there is evidence to indicate that an animal could be moved immediately to practically any task in the battery immediately after CHASE with minimal delays in learning.

\section{Would the Animals Show Interest in the Joystick?}

Substantial effort was invested into answering several questions. How might the animals be "magazine trained"? How can the rhesus be shaped most efficiently to touch the joystick? What measures are necessary to prevent responding from extinguishing? What potential distractions that might compete with joystick manipulation must be eliminated? Hindsight and experience now indicate that none of these issues represented serious threats to training.
Rhesus monkeys have learned to respond appropriately without autoshaping, magazine training, or experimentercontrolled (i.e., manual) reinforcement of selective behaviors. The animals trained to date have essentially been placed into position before the test stations and have trained themselves with minimal supervision. The rhesus exhibit an immediate curiosity about the joystick and, generally, an immediate appreciation of fruit-flavored pellets. Whereas early wisdom dictated that available food (e.g., food remaining from previous feedings) should be withdrawn before training, the actual data suggest that the animals work and learn despite the availability of other food, the presence of people or other animals, and access to a relatively large cage with other potential activities.

Each of our 10 rhesus monkeys performs $700-1,500$ trials per day, depending on task demands, task variety, and performance proficiency. Across the 4 years that Abel and Baker have been working on joystick-based tasks, this level of productivity has shown no enduring decrement. These observations suggest that the test system and tasks not only interest the rhesus, but that these monkeys maintain a high level of interest across long periods of time.

\section{How Important Would the Specific System Parameters Be for Performance?}

Although there are certainly some critical elements of the test system, many of the details of implementation appear to be flexible (at least with respect to testing behaviorally competent animals). These are summarized in Table 1. The central conclusion from these observations is that the joystick-related behaviors are robust and generalize well from task to task, from configuration to configuration, and from animal to animal.

Pellet parameters. Pellet size, for example, appears to bear little impact on learning and performance. Abel

Table 1

Parametric Investigations and Results

\begin{tabular}{|c|c|c|c|}
\hline Manipulation & Values & Productivity & Performance \\
\hline Monitor type & CRT, LCD & no effect & no effect, assuming fast LCD update rate \\
\hline Monitor size & $13,8,6$, and 4 in. & no effect & $\begin{array}{l}\text { small decrement in performance on difficult discriminations with } \\
\text { smallest monitors }\end{array}$ \\
\hline Joystick brand & $\begin{array}{l}\text { various, ranging in cost } \\
\text { from } \$ 9 \text { to } \$ 130\end{array}$ & no effect & no effect \\
\hline Joystick size & various & no effect & no effect \\
\hline Joystick orientation & $\begin{array}{l}\text { horizontal, vertical, and } \\
\text { intermediate angles }\end{array}$ & no effect & no effect, but untried with naive animals \\
\hline Sound feedback & yes, no & no effect & absence of sound feedback disrupts learning but not performance \\
\hline Speaker/amplifier & $\begin{array}{l}\text { Radio Shack Models } \\
32-2031 \mathrm{a} \text { and } 277-1008 \mathrm{c}\end{array}$ & no effect & no effect \\
\hline Input device & joystick, touch screen, button & \multicolumn{2}{|c|}{$\begin{array}{l}\text { limited evidence to suggest an advantage for using the joystick (ease of training, } \\
\text { use, availability of response topography, etc.) }\end{array}$} \\
\hline Color & monochrome, color & no effect & $\begin{array}{l}\text { small decrement in performance on difficult discriminations } \\
\text { without color }\end{array}$ \\
\hline Pellet size & $45,97,190$, and $300 \mathrm{mg}$ & no effect & no effect \\
\hline Pellet type & $\begin{array}{l}\text { Noyes, Bioserve } \\
\text { (various flavors) }\end{array}$ & no effect & no effect \\
\hline Subject age & $2-9$ years & no effect & no effect \\
\hline Computer type/speed & $\begin{array}{l}\mathrm{XT}: 8-10 \mathrm{MHz} \\
286,386: 12-16 \mathrm{MHz}\end{array}$ & no effect & faster cursor movement (thus response times) with faster processors \\
\hline
\end{tabular}


and Baker were trained with 45-mg pellets. When pellet size was shifted, or as subsequent animals were trained with 97-, 190-, or 300-mg pellets, no differences in productivity (number/rate of trials performed) or performance level were observed. For example, Abel and Baker performed 1,153 trials/day (receiving 1,074 pellets/day) in baseline testing with $97-\mathrm{mg}$ pellets. Upon their first experience with $300-\mathrm{mg}$ pellets, the animals performed an average of 1,092 trials/day ( 1,016 pellets/day). These differences were not statistically significant; neither did the percentage of trials completed successfully (i.e., that were rewarded) significantly differ between the conditions. This appears to be true across a range of pellet sizes, flavors, and vendors.

Joystick parameters. Similarly, testing with different joystick brands, sizes, and orientations has not been observed to influence performance or productivity. It is important to note, however, that the horizontal orientation of the joystick that we typically employ (i.e., with the handle pointing at the subject, so that cursor movements are isomorphic to joystick movements) may be important for establishing joystick manipulation skills in naive animals.

Monitor parameters. Because the software is CGAcompatible, graphics quality has not been a problem (except for the VGA-distance difficulty described below). We have tested animals with a variety of monitor types and sizes, including the 8-in. Sharp color LCD. Because stimulus size is proportional to monitor size, a small but significant decrement (about 8\%) in discrimination performance (e.g., learning set, matching to sample) has been observed with the use of 4-in. monitors, and there appears to be a transient compromise in pursuit tracking with the smallest sizes.

Color is greatly preferred, and matching-to-sample performance may be compromised $2 \%-20 \%$ when only monochromatic stimuli are employed (Washburn, 1990). However, productivity, psychomotor, and even learning measures show no disruption from the loss of this important stimulus dimension.

Sound. A large corpus of data supports the contention that behaviorally competent animals (i.e., those that have been trained on the tasks) can work without sound feedback without disruption. The exception to this rule consists of learning tasks, in which sound feedback provides a redundant cue (with pellet delivery) regarding response accuracy. Transfer index task performance is significantly better (6\%) with sound feedback, as is learning set performance $(7.5 \%)$. It is also anticipated that the availability of sound feedback is important to establishing requisite joystick skills, although this remains unexplored with naive animals.

The locus of sound feedback may also be important. For a variety of reasons, we strongly recommend externalizing the sound delivery through a speaker/amplifier. We have had good success with several models of amplifier. The procedure for modifyin ${ }_{c}$ the computer for this purposenot a simple rerouting of the sound signal directly to the external unit-is described elsewhere (Washburn, 1992b).
Computer parameters. The basic platform of the LRCCTS was, until very recently, an XT-compatible with CGA graphics. As technology advances, one must eventually decide to upgrade the quality of all hardware, and doing so has some cost. Because reading the joystick, moving the stimuli, and so forth are dependent on the cycling rate of the computer, some task measures such as response time will be different with the different computers. Adjusting cursor speed to make test systems comparable serves to make the movement fidelity different between different systems. The programmable game boards, so critical for reliable testing with faster computers, frequently serve to exacerbate this problem. However, it should be noted that the problem has not been prohibitive in our testing; it has merely required the collection of additional data and, at most, some scaling of normative curves.

Input device. We have primarily used joysticks as input devices, although at times we have used button panels for certain types of testing. We have tried using touch screens on only a couple of occasions with the monkeys. For several reasons, we think that joysticks are an ideal device. They are inexpensive, readily available, and, given the caveats below, fairly reliable. There is good evidence that training is quick and facile with them; a naive monkey quite naturally reaches out to explore them. Finally, cursor movement on a screen yields response topography data that cannot be obtained with touch screens or button-based devices. Such data may be crucial in the analysis of some types of scientific questions (see Washburn, 1992a).

\section{How Would This Testing Affect the Subjects' Psychological Well-Being?}

By ethical, scientific, and legal mandate, scientists must be concerned with the psychological well-being as well as the physical health of research populations. The testing paradigm described here was designed to promote and to provide a means for assessing this well-being. On the other hand, one might see 8- to 24-h/day testing as severe, perhaps even diminishing the well-being of research subjects. Notwithstanding, preliminary data are uniform in support of the enrichment value of the video-task testing paradigm (Washburn \& Rumbaugh, 1991). This evidence indicates that the animals are physically healthy when maintained as has been described above; that they are motivated to work, at least in part, by factors intrinsic to the tasks; that they thrive under conditions of control, challenge, and competition that the paradigm permits; and that they will choose to engage in task-related activity even in the presence of attractive other options (Washburn \& Rumbaugh, 1991).

\section{Protection of the Test System}

Although we anticipated the need to plan carefully and work energetically for the protection of electronic and electrical apparatus, experience suggests that this cannot be overemphasized. The probing fingers and prying hands 
of monkeys (and human subjects as well, one might add!) make security of the apparatus a significant challenge, and areas in which animals are maintained are frequently less than ideal for the use and storage of delicate electronic apparatus.

At our laboratory, we have experimented with many permutations of system configuration (e.g., the computer system secured against a wall and cages secured to the system; cages secured against a wall and the test system on a Lexan-encased cart secured to the cage; computers and monitors together within an enclosure; computers outside the room and monitors inside the room; etc.). The current configuration seems to work well. The monitor, dispenser, joystick, and speaker/amplifier are located on a wall-mounted stand within the test area, housed within a vented Lexan enclosure. All wires are run through plastic conduit to distant computers, which are located outside the test rooms. The cages are rolled up to the test stations and secured, so that the animals must reach through the mesh of their cages to have access to the system. Only the joystick handle and pellet cup are exposed to the animals' hands. This prevents the animals from gaining access to wires and equipment, protects the computers from fluids, debris, and vibrations, and affords easy access to the computers by experimenters without disturbing the rhesus.

One concern introduced by this configuration is distance. Painstaking testing was conducted to ensure that the CGA video, the joystick, the audio, and the reward delivery signals could traverse the 3-10 m of extended cable without disruption. However, as long as a single continuous extension cable (some commercially available, but most requiring basic wiring) is used between the computer and the remote peripheral, and assuming that the connections are good, no problems have been observed with the basic test configuration as a function of distance. The need for quality extension is particularly important for pellet delivery at a distance. Reliable CGA and EGA video, joystick readings, and audio appear to be produced irrespective of distance from the computer. However, systems with VGA graphics require a commercially available signal booster for the use of a monitor that is remote from the computer.

\section{Reliability of the Joystick}

As flexible, powerful, and affordable as joysticks are as input devices, they are clearly the weakest link in the testing system. Each consists of two potentiometers (one for each axis of movement), the analog values from which are converted to digital values within the range 0-255. Three related problems can be seen in the operation of analog joysticks. The first is calibration; a program must "know" what values are associated with no movement (i.e., the joystick's center position) in order to compute direction and degree of joystick movement. Although procedures can be implemented within software for establishing these values, the values may also drift or otherwise change within a test session. The second, related, problem is range; with use, the range and continuity of values returned by these potentiometers is reduced. Finally, there is the problem of noise. Untouched, a joystick may return multiple values within a small range; the software interprets this noise as movements even though none actually occur. As a joystick ceases to function properly, a variety of problems may be observed. Possible solutions to these problems are suggested in Table 2 .

Several steps can be taken to decrease the likelihood of these joystick difficulties. First, we calibrate the joystick at least daily. This involves observing the center and range values for each axis of movement. Second, we eliminate the "trim knobs" to prevent accidental adjustment of joystick calibration; these can be cut or filed so that they can only be moved with a screwdriver or similar tool. This is as important for testing human adults as it is for testing nonhuman primates; most of our testing problems with human subjects have involved manipulation (inadvertent or otherwise) of these dials. We now routinely use a hot soldering iron to "spot seal" a potentiometer "trim knob" in place; such a melted spot can be broken if the joystick must be manually recalibrated, but it is difficult to change accidentally. Third, we protect the joystick and wiring from bites, water, and electrical shorts. Fourth, we use a multispeed programmable game board to reduce noise. Fifth, we have implemented a "dead zone" parameter in each task, which requires movement to be greater than some value before it can result in cursor movement; in this way, the software can absorb some of the noise and drift associated with joystick performance. Finally, we secure the joystick firmly in position. Regardless of the species or age of the subject, the likelihood of joystick problems appears to increase with any potential movement of the device. Human subjects have, without fail, preferred to have joysticks unmounted for comfort, but have shown the highest levels of performance and reliability when the joysticks were stationary.

A final concern regarding the joystick is that it has numerous removable parts (e.g., the "fire" buttons, the plastic handle itself, etc.). Care must be used to ensure that these either remain on the joystick or are removed beforehand. No difficulties have been observed with human or nonhuman primates using only the metal shaft with no plastic handle or buttons.

With the implementation of these suggestions, the life and reliability of the average joystick has increased substantially. In 1987, joysticks were repaired or discarded and replaced (including replacement due to physical damage by the monkeys) every 28 days on the average (with a between-day variability in readings of over $40 \%$ ). In the subsequent 2 years, joysticks lasted an average of 122 days. In the period since, joysticks have lasted for an average of 9 months, and daily inspection of calibration reveals only a $3 \%$ difference in day-to-day center value readings over this period.

\section{Volume of Data}

Two other pleasant surprises were soon apparent. First, the animals learned the tasks more quickly than programmers could provide them. Thus, whereas Abel and Baker 
Table 2

Troubleshooting Joystick Problems

\begin{tabular}{|c|c|}
\hline Problem & Possible Solution \\
\hline Cursor moves with joystick static & $\begin{array}{l}\text { Increase "dead zone" } \\
\text { Increase speed of game card }\end{array}$ \\
\hline Cursor static when joystick moves & $\begin{array}{l}\text { Check joystick connection } \\
\text { Decrease "dead zone" } \\
\text { Decrease speed of game card }\end{array}$ \\
\hline Cursor moves faster in one direction than the other & $\begin{array}{l}\text { Check calibration of joystick } \\
\text { Ensure full range of joystick motion }\end{array}$ \\
\hline Cursor moves in opposite direction of joystick & $\begin{array}{l}\text { Check orientation of joystick } \\
\text { Check calibration of joystick } \\
\text { Replace joystick }\end{array}$ \\
\hline Cursor moves erratically or uncontrollably & $\begin{array}{l}\text { Decrease cursor speed } \\
\text { Check calibration } \\
\text { Ensure that connection is dry } \\
\text { Replace joystick }\end{array}$ \\
\hline Cursor moves too slowly & $\begin{array}{l}\text { Increase cursor speed } \\
\text { Decrease speed of game card } \\
\text { Decrease "dead zone" }\end{array}$ \\
\hline Joystick "center position" changes within a session & $\begin{array}{l}\text { Remove "trim knobs" } \\
\text { Adjust calibration each trial within the software } \\
\text { Replace joystick }\end{array}$ \\
\hline Joystick cannot be calibrated to center of range & $\begin{array}{l}\text { Open joystick base and manually turn potentiometer } \\
\text { while holding "trim knobs" still } \\
\text { Replace joystick }\end{array}$ \\
\hline
\end{tabular}

required several years to advance through the 18 tasks of the current battery, subsequent animals have done so in as few as 6, and more typically 12 , months. More important at this time, however, is the volume of data produced by this procedure. A single animal performing 1,000 trials per day can generate over a megabyte of summary data each week. If path data (or detail data; the $x, y$ coordinates of every target and cursor movement for each trial) are recorded, this volume of data can be increased more than 25 times! Thus, this very rich test paradigm must be accompanied by a careful data management plan for the retention, analysis, compression, and security of data.

\section{CONCLUSION}

In reviewing the effort and concern surrounding the design, inplementation, and development of the video-task testing paradigm, we have identified numerous suggestions and caveats. Having employed the test system with monkeys, apes, and humans, we can unreservedly endorse its use. The flexibility, affordability, and efficiency of the paradigm appear to be unrivaled and vastly outweigh the few problems and difficulties that it entails.

\section{REFERENCES}

Hopkins, W. D., Washburn, D. A., \& Rumbaugh, D. M. (1990). Processing of form stimuli presented unilaterally in humans, chimpanzees (Pan troglodytes), and monkeys (Macaca mulatta). Behavioral Neuroscience, 104, 577-582.

Meyer, D. R., Treichler, F. R., Meyer, P. M. (1965). Discretetrial training techniques and stimulus variables. In A. M. Schrier, H. F. Harlow, \& F. Stollnitz (Eds.), Behavior of nonhuman primates: Modern research trends (Vol. 1, pp. 1-49). New York: Academic Press.

Richardson, W. K., Washburn, D. A., Hopkins, W. D., Savage-
Rumbaugh, E. S., Rumbaugh, D. M. (1990). The NASA/LRC Computerized Test System. Behavior Research Methods, Instruments, \& Computers, 22, 127-131.

Rumbaugh, D. M., Richardson, W. K., Washburn, D. A., SavageRumbaugh, E. S., Hopkins, W. D. (1989). Rhesus monkeys (Macaca mulatta), video tasks, and implications for stimulus-response spatial contiguity. Joumal of Comparative Psychology, 103, 32-38. SAVAGE-Rumbaugh, E. S. (1986). Ape language: From conditioned response to symbol. New York: Columbia University Press.

Smith, S. T., JR., Cash, C., Barr, S. E., PUtney, R. T. (1986). The nonspeech assessment of hemispheric specialization in retarded children. Neuropsychologia, 24, 293-296.

STollNitz, F. (1965). Spatial variables, observing responses, and discrimination learning sets. Psychological Review, 72, 247-261.

WASHBURN, D. A. (1990). PC-compatible computer-generated stimuli for video-task testing. Behavior Research Methods, Instruments, \& Computers, 22, 132-135.

W ASHBURN, D. A. (1992a). Analyzing the path of responding in mazesolving and other tasks. Behavior Research Methods, Instruments, \& Computers, 24, 248-252.

WASHBURN, D. A. (1992b). External audio for IBM-compatible computers. Behavior Research Methods, Instruments, \& Computers, 24, 80-81.

Washburn, D. A., Hopkins, W. D., Rumbaugh, D. M. (1989). Video-task assessment of learning and memory in macaques (Macaca mulatta): Effects of stimulus movement upon performance. Journal of Experimental Psychology: Animal Behavior Processes, 15, 413-420.

Washburn, D. A., \& Rumbaugh, D. M. (1991, October). Investigations of rhesus monkey (Macaca mulatta) video-task performance: Evidence for enrichment. Paper presented to the annual meeting of the American Association for Laboratory Animal Science, Buffalo, NY.

\section{APPENDIX A}

\section{Materials List for Implementation of Video-Task Testing Paradigm}

1. IBM-compatible microcomputer

Minimum: XT-compatible, 8-MHz speed, 5.25-in. floppy, 20-MB hard drive, CGA color graphics board/monitor 
Recommended: 386SX-compatible, 16-MHz speed, 5.25/3.5-in. floppies, 40-MB hard drive, VGA color graphics board/monitor

\section{Joystick}

Minimum:

IBM-compatible analog joystick (any off-the-shelf brand seems to work fine)

Recommended: Kraft KC3 (820047-10)

3. Game board

Recommended: Kraft Multispeed Programmable Joystick adaptor card (IBM PC-compatible, two game ports) No. 820144

4. Pellet dispenser

Recommended: Gerbrands G5120 dispenser with ac Adapter G5160; this unit comes with 45-mg pellet size standard; for larger sizes (see below) one can also purchase a conversion kit

5. I/O

Recommended: Keithley/Metrabyte PIO-12 digital I/O interface and SRA-01 solid-state relay board (or ERA-01) with cable (C1800)

6. Pellets

Minimum:

Palatable 45-, 97-, 190-, or 300-mg pellets

Recommended: Noyes Formula L 97-mg pellets

7. Pellet receptacle

Minimum: Any sturdy receptacle should function

Recommended: Lab Products H-1377

8. External speaker/amplifier

Recommended: Radio Shack 32-2031 A with ac adapter

9. GSU/NASA Behavior/Performance Project Training Software

Recommended: Phase 3.3; contact the authors for details and availability

\section{Manufacturers}

Gerbrands Corp.

8 Beck Road

Arlington, MA 02174

(617) 648-6415

Keithley/Metrabyte

440 Myles Standish Blvd. P. J. Noyes Company, Inc.

Tauton, MA 02780

(508) $880-3000$

P. O. Box 381, Bridge St.

Lancaster, NH 03584

(800) LAB-CHOW

\section{APPENDIX B \\ Language Research Center Computerized Test System \\ Task Summary}

Psychomotor tasks, designed to provide a fine-grain assay of motor control and hand-eye coordination across selected types of tasks and levels of task difficulty:

SIDE: Move the cursor to stationary targets that are systematically varied in size and number. Dependent variables: movement times (MT), response times (RT), accuracy levels (error $=$ hitting nontarget wall), and path of responding. Potential independent/control variables: size/number of walls, speed of cursor, intertrial interval (ITI), correction procedure.

CHASE: Move the cursor to a dynamic/moving target that moves predictably or randomly. Dependent variables: MT, $\mathrm{RT}$, path of responding. Potential independent/control variables: target path, target speed, cursor speed, target image, ITI, fixed ratio (FR) size, reward type/line, sound feedback with cursor movement, movement of target with cursor movement (freeze frame).

PURSUIT: Maintain continuous contact with a target (pursuit tracking) that moves either predictably or randomly. Dependent variables: MT, RT, accuracy (errors = moving off target), path of responding. Potential independent/control variables: target path, pursuit duration, collision distance, cursor/target speed, target image, ITI, FR size, reward type/line, sound feedback.

Perception and prediction tasks, designed to measure an animal's perception and acuity for visual and auditory stimuli, time, and orientation, and to determine how this information is utilized in responding:

LASER: Issue a "shot" from a stationary turret at a trajectory that will intersect with the movement of a target. Dependent variables: MT, RT, number of shots, number of aborted shots. Potential independent/control variables: shot speed, target path, "shot freeze," FR size.

BLACK HOLE: Catch a target that moves through and may disappear within a midscreen barrier, through which the cursor cannot traverse. Dependent variables: MT, RT, number of times the target passes through circle, response path/ difference value. Potential independent/control variables: target path, target visible versus invisible within hole, target speed, stop when hitting wall.

MTS (matching to sample): A baseline measure of visual discrimination and acuity is obtained. A sample stimulus is presented on the screen, followed by two choice stimuli; the subject must touch the choice stimulus that matches the sample. Dependent variables: MT, RT, accuracy (error $=$ touching nonmatching stimulus). Potential independent/control variables: target characteristics (form, size, color), target movement, FR size, ITI.

SDI: Sameness-difference discriminations provide an alternative to MTS for assessing visual discriminations and reten-

450 W. California Ave.

Vista, CA 92083 
tion; subjects match to sample on same trials but contact a " $D$ '" on different trials. Dependent variables: MT, RT, accuracy (error $=$ " $D$ " on same or matching on different). Potential independent/control variables: target characteristics, target movement.

DVF: A measure of perceptual threshold, the amount of time during which a stimulus must be exposed in order for it to be recognized, is obtained for each of the cerebral hemispheres. The task requires contact with a circle, positioned midscreen, followed by a brief stimulus "flash" either to the left or right; the subject must then match to sample. Dependent variables: MT, RT, accuracy. Potential independent/control variables: target characteristics, visual field of presentation, presentation duration.

DETECT: Stimuli are presented, at random points in time, amid a field of noise; subjects respond in a go/no-go paradigm, holding the joystick down until the signal (sample) is seen. Dependent yariables: RT, accuracy (error = failing to respond to a signal, a miss, or responding in the absence of a signal, a false alarm). Potential independent/control variables: target characteristics (form, color, size), vigilance interval, response window.

Learning and retention tasks, designed to measure the capacity of an organism to learn, to retain, and to transfer information:

TI: The transfer index paradigm was developed to provide a single measure of learning and the ability to transfer learning. Two-choice discrimination learning to a criterion is followed by reversal testing; in addition, a series of problems can be presented, each for six trials, for learning set testing. Dependent variables: MT, RT, accuracy (pre- and postreversal; error $=$ selecting negative stimulus). Potential independent/control variables: criterion level, target characteristics, target movement, ITI, FR size.

MEDIATION: The mediational paradigm is a variant of the TI procedure that provides specific information about how an organism is learning (i.e., via associative or mediational strategies). Dependent variables: MT, RT, accuracy. Potential independent/control variables: mediation condition (A-B+, $\mathrm{C}-\mathrm{B}+, \mathrm{A}-\mathrm{C}+$ ), target characteristics, target movement, ITI, FR size.

MAZE: Subjects must navigate familiar or novel mazes in this test for spatial learning, transfer, and long-term retention. Dependent variables: MT, RT, accuracy (error = wrong alley in the maze), response path. Potential independent/ control variables: maze complexity, cursor speed, target movement, maze familiarity (e.g., new vs. learned mazes, learned vs. mirror-imaged mazes), retention interval.

LTM: Long-term memory is tested by examining the savings in learning associated with overlearned and unfamiliar discrimination problems. Dependent variables: MT, RT, accuracy, savings. Potential independent/control variables: familiarity of problems (experience/practice, retention interval).

Working memory tasks, designed to measure the degree to which short-term memory for visual and spatial stimuli is functioning efficiently, and to determine the extent that deficits in task performance might be attributed to failure to use or to retain information over relatively brief temporal periods:

DMTS: A primary test for short-term memory testing is the delayed matching-to-sample paradigm; a retention interval of some length is introduced between removal of the sample and presentation of the choice stimuli. Dependent variables: MT, RT, accuracy. Potential independent/control variables: target characteristics, target movement, retention interval.

Delayed responding: Delayed responding is an alternate paradigm for assaying the function of visual working memory, which allows corroboration of the STM data and one measure of cerebral frontal lobe function. Subjects touch a flashing stimulus; following a retention interval, they must identify this sample from two choice stimuli. Dependent variables: RT, accuracy. Potential independent/control variables: response cue (form vs. spatial), target characteristics.

Enrichment tasks, designed to provide each subject with the freedom to exercise control over activities and, ultimately, to assay each subject's preference for tasks or options and to demonstrate the relation between performance on tasks and psychological well-being:

SELECT: A menu format in which any task from a pool of available activities can be selected. Preference data and latency data are used to examine willingness/readiness to work; in addition, the SELECT format subsumes all other tasks, in that the data provided by each individual task will also be obtained when selected in this paradigm. Dependent variables: task preferences, MT, RT. Potential independent/control variables: available options; individual task variables.

NUMBER: Quantities of numerals are presented, the selection of one of which results in the corresponding number of pellets' being dispensed. Because the animal is rewarded irrespectively of the numeral selected (only the relative number of pellets differentiates the choices), this allows a paradigm for assaying choice behavior in a "no-lose" situation; however, the choice data and particularly the RTs are very revealing with respect to the organism's well-being and cognitive functioning. Dependent variables: MT, RT, accuracy (error $=$ not selecting largest available numeral). Independent variable: available numerals.

COMPETE: The LASER task described previously is extended to a familiar setting in which each subject must compete against the computer for rewards; the COMPETE task, as measured by number of shots and RT, appears to tap motivational levels and potentially social constructs. Dependent variables: see LASER. Potential independent/control variables: contested vs. uncontested; see LASER. 